\title{
The structure of radiative shock waves
}

\section{Effects of electron thermal conduction}

\author{
Yu. A. Fadeyev ${ }^{1}$, H. Le Coroller ${ }^{2}$, and D. Gillet ${ }^{2}$ \\ 1 Institute for Astronomy of the Russian Academy of Sciences, Pyatnitskaya 48, 109017 Moscow, Russia \\ 2 Observatoire de Haute-Provence - CNRS, 04870 Saint-Michel l'Observatoire, France
}

Received 12 September 2001 / Accepted 11 June 2002

\begin{abstract}
We consider the structure of steady-state radiative shock waves propagating in partially ionized hydrogen gas with density $\rho_{1}=10^{-10} \mathrm{gm} \mathrm{cm}^{-3}$ and temperature $3000 \mathrm{~K} \leq T_{1} \leq 8000 \mathrm{~K}$. The radiative shock wave models with electron thermal conduction in the vicinity of the viscous jump are compared with pure radiative models. The threshold shock wave velocity above which effects of electron thermal conduction become perceptible is found to be $U_{1}^{*} \approx 70 \mathrm{~km} \mathrm{~s}^{-1}$ and corresponds to the upstream Mach numbers from $M_{1} \approx 6$ at $T_{1}=8000 \mathrm{~K}$ to $M_{1} \approx 11$ at $T_{1}=3000 \mathrm{~K}$. In shocks with efficient electron heat conduction more than a half of the hydrogen atoms are ionized in the radiative precursor, whereas behind the viscous jump the hydrogen gas undergoes the full ionization. The existence of the electron heat conduction precursor leads to the enhancement of the Lyman continuum flux trapped in the surroundings of the discontinuous jump. As a result, the partially ionized hydrogen gas of the radiative precursor undergoes an additional ionization $\left(\delta x_{\mathrm{H}} \lesssim 5 \%\right)$, whereas the total radiave flux emerging from the shock wave increases by $10 \% \leq \delta\left(\boldsymbol{F}_{\mathrm{R}}\right) \leq 25 \%$ for $70 \mathrm{~km} \mathrm{~s}^{-1} \leq U_{1} \leq 85 \mathrm{~km} \mathrm{~s}^{-1}$.
\end{abstract}

Key words. shock waves - hydrodynamics - radiative transfer - stellar atmospheres

\section{Introduction}

In our previous papers (Fadeyev \& Gillet 1998, 2000, 2001), hereinafter referred to as Papers I-III, we presented studies of steady-state radiative shock waves propagating in the partially ionized hydrogen gas with properties that are typical of atmospheres of pulsating late-type stars. The models were considered in terms of the self-consistent solution of the equations of fluid dynamics, radiation transfer and rate equations for the hydrogen atom and provide the reliable estimates of radiative energy losses of the shock wave. In these studies we adopted that at the viscous jump, which is treated as an infinitesimally thin discontinuity, the electron gas compresses adiabatically. Such an assumption is justified by the very low rate of energy exchange between protons and electrons within the viscous jump and thereby allows us to determine the postshock electron temperature from the simple adiabatic relation. However, it is known that the characteristic length scale of electron thermal conduction might be comparable with the thickness of the postshock relaxation zone (the length of the temperature equilibration zone) and, therefore, might affect perceptibly the spatial distribution of hydrodynamic variables at least in the vicinity of the viscous jump (see, for discussion, Zel'dovich \& Raizer 1967; Mihalas \& Weibel Mihalas 1984).

Effects of electron thermal conduction in two-temperature shock waves propagating in partially ionized helium and argon

Send offprint requests to: D. Gillet, e-mail: gillet@obs-hp. fr with temperatures and densities close to those of stellar atmospheres were investigated using both hydrodynamic and kinetic approaches by Grewal \& Talbot (1963), Jaffrin (1965), Lu \& Huang (1974). Solution of the more general problem involving the radiation transfer was considered by Vinolo \& Clarke (1973). The conspicuous result of these calculations is that the authors demonstrated the existence of the zone of the elevated electron temperature ahead of the viscous jump appearing due to the high thermal conductivity of the electron gas. For the hydrogen gas effects of electron thermal conduction were considered only in the limit of full ionization at Mach numbers as high as $M_{1} \approx 8$ (Vidal et al. 1993, 1995). This study, unfortunately, was confined to the problem of inertial confinement fusion for extremely high temperature and number density of electrons $\left(T=10^{8} \mathrm{~K}, n_{\mathrm{e}}=10^{22} \mathrm{~cm}^{-3}\right)$.

Now, the scarce studies of astrophysical radiative shock waves with electron thermal conduction in two-temperature gases are confined to the interstellar medium (Borkowski et al. 1989; Borkowski \& Shull 1990) and accreting white dwarfs (Imamura et al. 1987). In these works the heat conduction was found to substantially affect the structure of the postshock relaxation zone for shocks with velocities ranging from $70 \mathrm{~km} \mathrm{~s}^{-1}$ to $170 \mathrm{~km} \mathrm{~s}^{-1}$. In atmospheres of late-type pulsating stars the gas density is many orders of magnitude higher in comparison with that of the interstellar medium, whereas the velocities of shocks do not exceed $100 \mathrm{~km} \mathrm{~s}^{-1}$, so that the role of electron heat conduction in radiative losses remains highly 
uncertain. In this paper we compute the models of steady-state radiative shock waves propagating through the partially ionized hydrogen gas with properties typical for atmospheres of pulsating late-type stars and compare the pure radiative shock wave models with those in which electron thermal conduction is taken into account.

As in our previous papers we assume that the ambient unperturbed medium is homogeneous and effects of magnetic fields are negligible. Indeed, the magnetic field becomes important when the electron mean free path $l_{\mathrm{e}}$ is larger than the gyromagnetic radius $r_{\mathrm{B}}$. For the gas density considered in our study $\left(\rho=10^{-10} \mathrm{gm} \mathrm{cm}^{-3}\right)$ the condition $l_{\mathrm{e}} \gg r_{\mathrm{B}}$ fulfills for $B \gg 3$ Gauss. Furthermore, the magnetic pressure $B^{2} / 8 \pi$ becomes comparable with the gas pressure for $B>25$ Gauss. On the other hand, the strength of the magnetic field in late-type giants is $B \ll 1$ Gauss (Belvedere et al. 1982). Thus, effects of magnetic fields can be ignored without any loss of accuracy. We expect that results of our calculations can be applied to shock phenomena observed in atmospheres of radially pulsating giants and supergiants such as W Vir, RV Tau and Mira type variables.

In order to specify the model we use three parameters determining the structure of the steady-state radiative shock wave. These are the density $\rho_{1}$ and the temperature $T_{1}$ of the ambient hydrogen gas and the upstream gas flow velocity $U_{1}$.

\section{Equations for thermal conduction}

Detailed discussion of the basic equations used for calculation of the structure of radiative shock waves is given in our earlier papers and in this section we only consider the equations involving the terms responsible for electron thermal conduction. Equation (20) of Paper II describes the change of the translational specific energy of the electron gas

$\widetilde{E}_{\mathrm{e}}=\frac{3}{2} \frac{n_{\mathrm{e}}}{\rho} k T_{\mathrm{e}}$

and is written now as

$$
\begin{aligned}
\frac{\mathrm{d} \widetilde{E}_{\mathrm{e}}}{\mathrm{d} t}= & -P_{\mathrm{e}} \frac{\mathrm{d} V}{\mathrm{~d} t}+Q_{\mathrm{elc}}-\frac{\mathrm{d} \widetilde{E}_{\mathrm{I}}}{\mathrm{d} t}-\frac{\mathrm{d} \widetilde{E}_{\mathrm{ex}}}{\mathrm{d} t}-\frac{1}{\rho} \frac{\mathrm{d} E_{\mathrm{R}}}{\mathrm{d} t} \\
& -\frac{1}{\rho} \nabla \cdot \boldsymbol{F}_{\mathrm{R}}-\frac{1}{\rho} \nabla \cdot \boldsymbol{F}_{\mathrm{e}} .
\end{aligned}
$$

Here $n_{\mathrm{e}}$ is the number density of free electrons, $P_{\mathrm{e}}=n_{\mathrm{e}} k T_{\mathrm{e}}$ is the partial pressure of the electron gas, $V=1 / \rho$ is the specific volume, $Q_{\text {elc }}$ is the rate of energy gain by electrons in elastic collisions with neutral hydrogen atoms and hydrogen ions, $\widetilde{E}_{\mathrm{I}}$ and $\widetilde{E}_{\mathrm{ex}}$ are the ionization energy and the excitation energy per unit mass, $E_{\mathrm{R}}$ and $\boldsymbol{F}_{\mathrm{R}}$ are the radiation energy density and the total radiation flux, respectively,

$$
\boldsymbol{F}_{\mathrm{e}}=-K_{\mathrm{e}} \nabla T_{\mathrm{e}}
$$

is the electron heat conductive flux. The thermal conductivity $K_{\mathrm{e}}$ was calculated using the fitting formulae (Nowak \& Ulmschneider 1977) taking into account effects of partial ionization and converging with those given by Spitzer (1962) for the fully ionized hydrogen.
Equation (3) is valid until the mean free path of electrons is smaller than the characteristic temperature scale and for the too steep temperature gradient it becomes inapplicable due to effects of saturation of the heat flux. According to Cowie \& McKee (1977) the saturated heat flux $q_{\text {sat }}$ is proportional to the product of the thermal energy of electrons and their mean thermal velocity, that is,

$q_{\mathrm{sat}}=f\left(\frac{2 k T_{\mathrm{e}}}{\pi m_{\mathrm{e}}}\right)^{1 / 2} n_{\mathrm{e}} k T_{\mathrm{e}}$.

Unfortunately, the value of the flux limit factor $f$ for the partially ionized hydrogen gas is uncertain. Cowie \& McKee (1977) adopted $f=0.4$, whereas Borkowski et al. (1989) presented arguments in favour of $f \sim 0.1$. According to more recent kinetic calculations (Vidal et al. 1995) the flux limit factor is $f \approx 0.3$ and it is the value that we used in our study.

The presence of the divergence of the conductive flux in Eq. (2) increases the order of this equation and leads to the much stronger sensitivity of the resulting system of ordinary differential equations (see Eqs. (17)-(21) of Paper II) to the rounding-off errors. To keep the first order of Eq. (2) we calculated $\nabla \cdot \boldsymbol{F}_{\mathrm{e}}$ in each iteration together with the solution of the radiation transfer equation for the given spatial distribution of hydrodynamic variables. For the first iteration we replaced the spatial distribution of the electron temperature $T_{\mathrm{e}}$ obtained from the pure radiative model by the arbitrary function smoothing the run of $T_{\mathrm{e}}$ within $\sim 100 \mathrm{~cm}$ in both sides of the discontinuous jump. After the series of trial calculations we found that the final solution does not depend on the initial distribution of $T_{\mathrm{e}}$ since the region of the effective energy transfer by electron thermal conduction is by an order of magnitude narrower. The shock wave models were represented by $2000 \leq N \leq 8000$ cells with $\sim 10^{3}$ cells within the thermal conduction region.

The divergence of the conductive flux was computed using the second-order finite-difference formula

$$
\begin{aligned}
\left(\nabla \cdot \boldsymbol{F}_{\mathrm{e}}\right)_{j-1 / 2}= & -\frac{K_{\mathrm{e} j-1}}{\Delta X_{j-1}} \frac{T_{\mathrm{e} j-3 / 2}}{\Delta X_{j-1 / 2}} \\
& +\left(\frac{K_{\mathrm{e} j-1}}{\Delta X_{j-1}}+\frac{K_{\mathrm{e} j}}{\Delta X_{j}}\right) \frac{T_{\mathrm{e} j-1 / 2}}{\Delta X_{j-1 / 2}}-\frac{K_{\mathrm{e} j}}{\Delta X_{j}} \frac{T_{\mathrm{e} j+1 / 2}}{\Delta X_{j-1 / 2}},(5)
\end{aligned}
$$

where $\Delta X_{j}=\frac{1}{2}\left(\Delta X_{j-1 / 2}+\Delta X_{j+1 / 2}\right)$ and $\Delta X_{j-1 / 2}=X_{j}-X_{j-1}$ are the space intervals. All thermodynamic quantities are defined at cell centers having half-integer subscripts and the conductivity coefficient at cell interfaces is determined as the mass-weighted average:

$K_{\mathrm{e} j}=\frac{K_{\mathrm{e} j-1 / 2} \Delta m_{j-1 / 2}+K_{\mathrm{e} j+1 / 2} \Delta m_{j+1 / 2}}{\Delta m_{j-1 / 2}+\Delta m_{j+1 / 2}}$,

where $\Delta m_{j-1 / 2}=\rho_{j-1 / 2} \Delta X_{j-1 / 2}$ is the mass contained in the cell $\left[X_{j-1}, X_{j}\right]$. Thus, the divergence of the conductive flux in Eq. (2) was considered explicitly and during integration of the ordinary differential equations was calculated using the nonlinear interpolation.

The discontinuity is assumed to be located at $X_{J}=0$ and coordinates of cell centers just ahead of and just behind the discontinuity are denoted as $X_{J-1 / 2}$ and $X_{J+1 / 2}$, respectively. 
For the sake of convenience the quantities defined at these cell centers are denoted by superscripts minus and plus, respectively. In all models considered in our study the size of the central cell was set equal to $\Delta X_{J}=X_{J+1 / 2}-X_{J-1 / 2}=0.1 \mathrm{~cm}$.

Solution of the preshock initial-value problem is ended at the $(J-1 / 2)$ th cell center and in order to obtain the initial conditions for the postshock integration we use the RankineHugoniot equations relating quantities at both sides of the discontinuity at cell centers $X_{J-1 / 2}$ and $X_{J+1 / 2}$. For the radiative shock wave with electron heat conduction the RankineHugoniot equations are written as

$\rho U=C_{0} \equiv \dot{m}$,

$\dot{m} U+P_{\mathrm{g}}+P_{\mathrm{R}}=C_{1}$,

$\frac{1}{2} \dot{m} U^{2}+\dot{m} h+\boldsymbol{F}_{\mathrm{e}}+\boldsymbol{F}_{\mathrm{R}}+U\left(E_{\mathrm{R}}+P_{\mathrm{R}}\right)=C_{2}$,

where $h$ is the specific enthalpy (see Eq. (7) of Paper II), $P_{\mathrm{g}}$ is the gas pressure, $P_{\mathrm{R}}$ is the radiation pressure, $C_{0}, C_{1}$ and $C_{2}$ are the mass, momentum and energy fluxes. Solution of the Rankine-Hugoniot relations gives the compression ratio across the discontinuous jump $\eta=\rho^{+} / \rho^{-}$as the root of the quadratic equation

$A \eta^{2}-B \eta+C=0$

where

$$
\begin{aligned}
A= & \left(a_{\mathrm{T}}^{-}\right)^{2}+\frac{1}{5}\left(U^{-}\right)^{2}-\frac{2}{5} \frac{\boldsymbol{F}_{\mathrm{e}}^{+}+\boldsymbol{F}_{\mathrm{R}}^{+}-\boldsymbol{F}_{\mathrm{e}}^{-}-\boldsymbol{F}_{\mathrm{R}}^{-}}{\dot{m}} \\
& +\frac{2}{5} \frac{E_{\mathrm{R}}^{-}+P_{\mathrm{R}}^{-}}{\rho^{-}}, \\
B= & \left(a_{\mathrm{T}}^{-}\right)^{2}+\left(U^{-}\right)^{2}+\frac{2}{5} \frac{E_{\mathrm{R}}^{+}+P_{\mathrm{R}}^{+}}{\rho^{-}}-\frac{P_{\mathrm{R}}^{+}-P_{\mathrm{R}}^{-}}{\rho^{-}}, \\
C= & \frac{4}{5}\left(U^{-}\right)^{2},
\end{aligned}
$$

$a_{\mathrm{T}}=\left(P_{\mathrm{g}} / \rho\right)^{1 / 2}$ is the isothermal sound speed.

In contrast to our earlier studies where we assumed that the electron gas compresses at the viscous jump adiabatically in the present paper we employed the jump condition for the electron temperature written as

$T_{\mathrm{e}}^{+}=T_{\mathrm{e}}^{-}-\frac{\boldsymbol{F}_{\mathrm{e} J}}{K_{\mathrm{e} J}} \Delta X_{J}$

The postshock temperature of heavy particles is obtained from solution of Eqs. (7)-(9) and is given by

$$
\begin{aligned}
T_{\mathrm{a}}^{+}= & T_{\mathrm{a}}^{-}-\frac{n_{\mathrm{e}}^{-}}{n_{\mathrm{H}}^{-}}\left(T_{\mathrm{e}}^{+}-T_{\mathrm{e}}^{-}\right)+\frac{1}{5} \frac{\dot{m} U^{-}}{n_{\mathrm{H}}^{-} k} \frac{\eta^{2}-1}{\eta^{2}} \\
& -\frac{2}{5} \frac{\boldsymbol{F}_{\mathrm{e}}^{+}+\boldsymbol{F}_{\mathrm{R}}^{+}-\boldsymbol{F}_{\mathrm{e}}^{-}-\boldsymbol{F}_{\mathrm{R}}^{-}}{n_{\mathrm{H}}^{-} k U^{-}} \\
& -\frac{2}{5} \frac{\left(E_{\mathrm{R}}^{+}+P_{\mathrm{R}}^{+}\right) \eta^{-1}-\left(E_{\mathrm{R}}^{-}+P_{\mathrm{R}}^{-}\right)}{n_{\mathrm{H}}^{-} k}
\end{aligned}
$$

where $n_{\mathrm{H}}$ is the number density of hydrogen atoms.

\section{The structure of shocks with heat conduction}

In solution of the initial-value problem for the fluid dynamics and rate equations we considered the system of ordinary differential equations described in Paper II. The only exception is that within the interval $X_{\mathrm{ca}}=-100 \mathrm{~cm} \leq X \leq X_{\mathrm{cb}}=$ $100 \mathrm{~cm}$ surrounding the discontinuous jump the equation for $E_{\mathrm{e}}$ (Eq. (20) of Paper II) is replaced by Eq. (2). The large size of the interval with heat conduction taken into account was used in order to diminish the role of boundary conditions $\boldsymbol{F}_{\mathrm{e}}\left(X_{\mathrm{ca}}\right)=\boldsymbol{F}_{\mathrm{e}}\left(X_{\mathrm{cb}}\right)=0$ in calculations of the divergence of conductive flux $\nabla \cdot \boldsymbol{F}_{\mathrm{e}}$.

The vicinity of the discontinuous jump of the shock wave model with $\rho_{1}=10^{-10} \mathrm{gm} \mathrm{cm}^{-3}, T_{1}=3000 \mathrm{~K}$ and $U_{1}=$ $75 \mathrm{~km} \mathrm{~s}^{-1}$ is shown in Fig. 1 where as a function of $X$ are plotted the electron temperature $T_{\mathrm{e}}$ and the temperature of heavy particles $T_{\mathrm{a}}$ (upper panel), the heat conductive flux $\boldsymbol{F}_{\mathrm{e}}$, the Lyman continuum flux $F_{\text {Ly }}$ and the saturated heat flux $q_{\text {sat }}$ (middle panel), the divergence of the heat conductive flux $\nabla \cdot \boldsymbol{F}_{\mathrm{e}}$ and the divergence of the total radiative flux $\nabla \cdot \boldsymbol{F}_{\mathrm{R}}$ (lower panel). It should be noted that in the framework of our model the space coordinate $X$ increases while the gas element passes through the shock wave and in such a notation the upstream and downstream fluxes are negative and positive, respectively.

In the unperturbed hydrogen gas with density $\rho_{1}=$ $10^{-10} \mathrm{gm} \mathrm{cm}^{-3}$ and the temperature of $3000 \mathrm{~K} \leq T_{1} \leq 6000 \mathrm{~K}$ the length of the radiative precursor is $\sim 10^{4} \mathrm{~cm}$ and at the upstream gas flow velocity $U_{1}=75 \mathrm{~km} \mathrm{~s}^{-1}$ the temperature growth due to absorption of the Lyman continuum radiation emerging from the postshock region is $(\Delta T)_{\mathrm{r}} \approx 2000 \mathrm{~K}$. As is seen from Fig. 1 the length of the region of efficient heat conduction surrounding the discontinuous jump is of $\sim 10 \mathrm{~cm}$. Within such a narrow interval the divergence of the heat conductive flux exceeds the divergence of radiative flux by almost two orders of magnitude, so that the heating and cooling of gas are due to the electron heat conduction and the role of radiation is negligible.

For the model represented in Fig. 1 the electron temperature growth within the conductive precursor is of $\left(\Delta T_{\mathrm{e}}\right)_{\mathrm{c}} \approx$ $3 \times 10^{4} \mathrm{~K}$. A small fraction of the translational energy of the electron gas is lost in elastic collisions with hydrogen ions and the temperature of heavy particles increases within the conductive precursor by $\left(\Delta T_{\mathrm{a}}\right)_{\mathrm{c}} \approx 2000 \mathrm{~K}$.

The heat conductive flux $\boldsymbol{F}_{\mathrm{e}}$ does not exceed the total radiative flux and its maximum value is only comparable with the Lyman flux $F_{\text {Ly }}$ (see the middle panel of Fig. 1) which is of $\approx 40$ percent of the total radiative flux $\boldsymbol{F}_{\mathrm{R}}$. As is seen from Fig. 1 effects of saturation can play a perceptible role only in the conductive precursor just ahead of the discontinuous jump, whereas behind the discontinuity $q_{\text {sat }} \gg\left|\boldsymbol{F}_{\mathrm{e}}\right|$ because of the higher density and higher temperature of the gas.

The growth of the preshock electron temperature due to conductive heating is accompanied by increasing gas density. For example, in the pure radiative model with $\rho_{1}=$ $10^{-10} \mathrm{gm} \mathrm{cm}^{-3}, T_{1}=3000 \mathrm{~K}$ and $U_{1}=75 \mathrm{~km} \mathrm{~s}^{-1}$ the preshock compression ratio is $\left(\rho^{-} / \rho_{1}\right)_{\mathrm{r}}=1.008$, whereas in the model with heat conduction the preshock compression ratio is $\left(\rho^{-} / \rho_{1}\right)_{\mathrm{c}}=1.04$. The difference in compression ratios becomes 


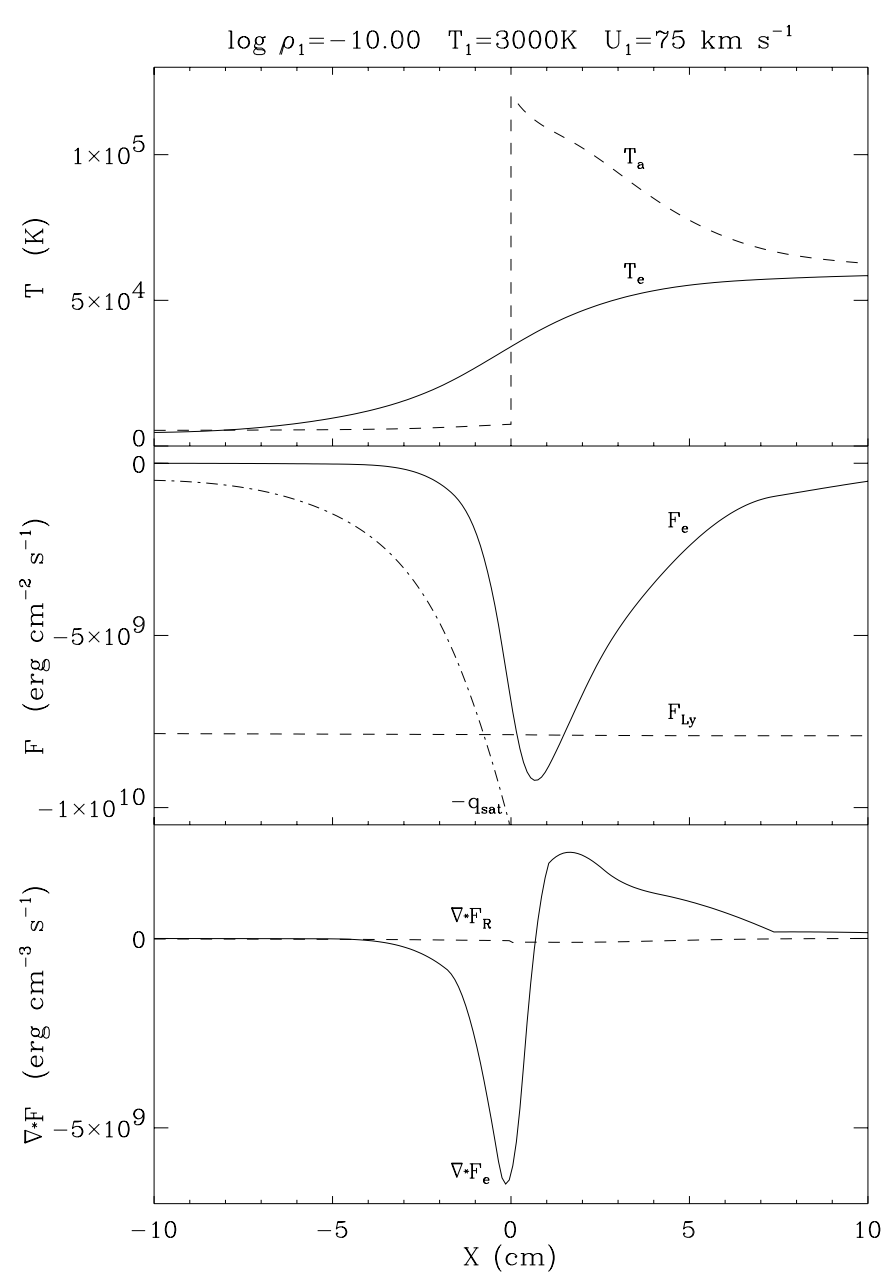

Fig. 1. The vicinity of the discontinuous jump located at $X=0$ of the shock wave model with $\rho_{1}=10^{-10} \mathrm{gm} \mathrm{cm}^{-3}, T_{1}=3000 \mathrm{~K}$ and $U_{1}=75 \mathrm{~km} \mathrm{~s}^{-1}$. Upper panel: the electron temperature $T_{\mathrm{e}}$ (solid line) and the temperature of heavy particles $T_{\mathrm{a}}$ (dashed line). Middle panel: the heat conductive flux $\boldsymbol{F}_{\mathrm{e}}$ (solid line), the Lyman continuum flux $F_{\mathrm{Ly}}$ (dashed line )and the saturated heat flux $q_{\text {sat }}$ (dot-dashed line). Lower panel: the divergence of the heat conductive flux $\nabla \cdot \boldsymbol{F}_{\mathrm{e}}$ (solid line) and the divergence of the total radiative flux $\nabla \cdot \boldsymbol{F}_{\mathrm{R}}$ (dashed line).

more significant in the postshock region and increases with increasing distance from the discontinuous jump. For example, at the boundary of the thermalization zone $(\log X \approx 1)$ the ratio of gas densities evaluated for the conductive and pure radiative models is $\rho_{\mathrm{c}} / \rho_{\mathrm{r}} \approx 1.1$, whereas at the boundary of the recombination zone $(\log X \approx 4.5)$ this ratio is as high as $\rho_{\mathrm{c}} / \rho_{\mathrm{r}} \approx 1.6$. Two plots of the postshock compression ratios $\rho / \rho_{1}$ for the conductive and radiative shock wave models are shown in Fig. 2.

The stronger compression in shock waves with heat conduction implies that relaxation processes are somewhat faster in comparison with those in pure radiative shocks. Such a difference is due to the fact that existence of the preshock conductive precursor leads to the elevation of the radiation energy density within the whole area of the trapped Lyman continuum radiation. This area is confined by the layers of hydrogen ionization in the preshock region (the radiative precursor) and by the layers of hydrogen recombination behind the discontinuous jump. The enhancement of the radiation energy density is

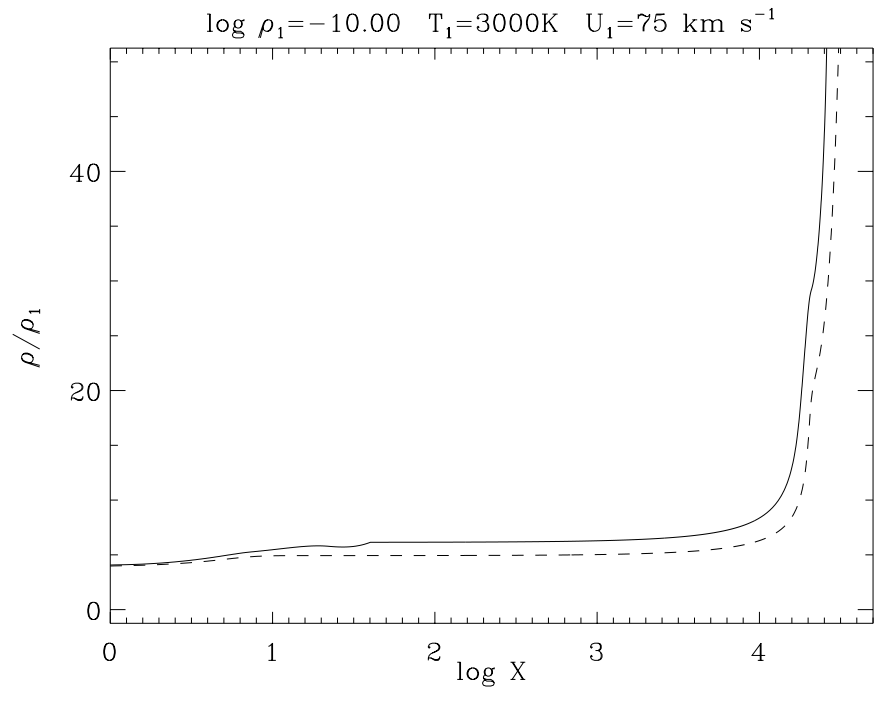

Fig. 2. The postshock compression ratio $\rho / \rho_{1}$ in the shock wave model with electron heat conduction (solid line) and in the pure radiative model (dashed line).

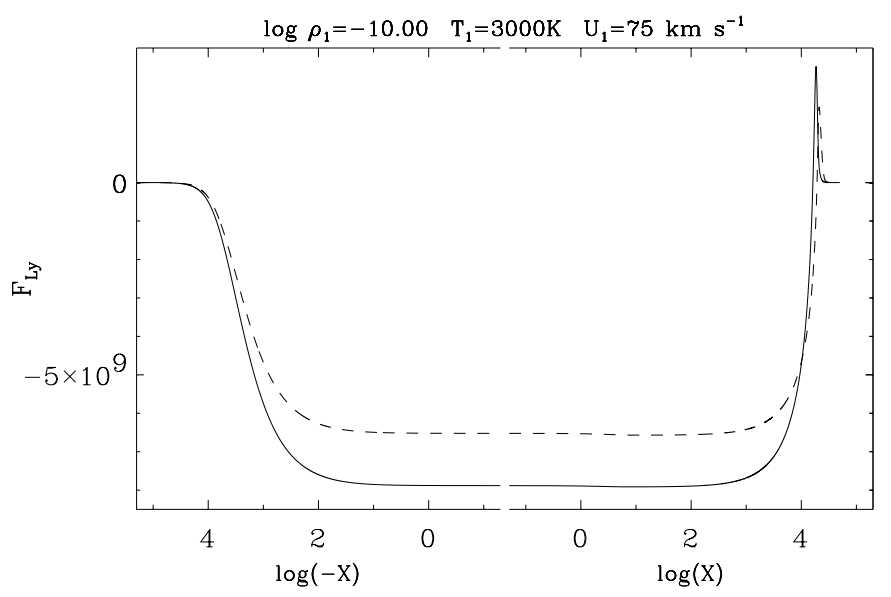

Fig. 3. The Lyman continuum flux in the conductive (solid line) and pure radiative (dashed line) shock waves. The left and the right panels represent the preshock and postshock regions, respectively.

illustrated in Fig. 3 where we plot the Lyman continuum flux as a fuction of $X$ for pure radiative and for the conductive model. For the sake of convenience we use the logarithmic scales for the both preshock and postshock regions that are represented by the left and by the right panels, respectively.

As was shown in our earlier papers (see, for example, Papers I and II) the populations of atomic levels are governed by radiative transitions because the rates of corresponding collisional transitions are several orders of magnitude smaller throughout the whole shock wave. Thus, elevation of the radiation energy density in the Lyman continuum leads to the larger degree of preshock hydrogen ionization and at the same time to the faster ionization (if the hydrogen gas is still partially ionized in the radiative precursor) in the postshock region. This effect is illustrated in Fig. 4 representing the spatial distributions of the electron temperature $T_{\mathrm{e}}$ and the hydrogen ionization degree $x_{\mathrm{H}}$ for the both conductive and pure radiative shock wave models. 


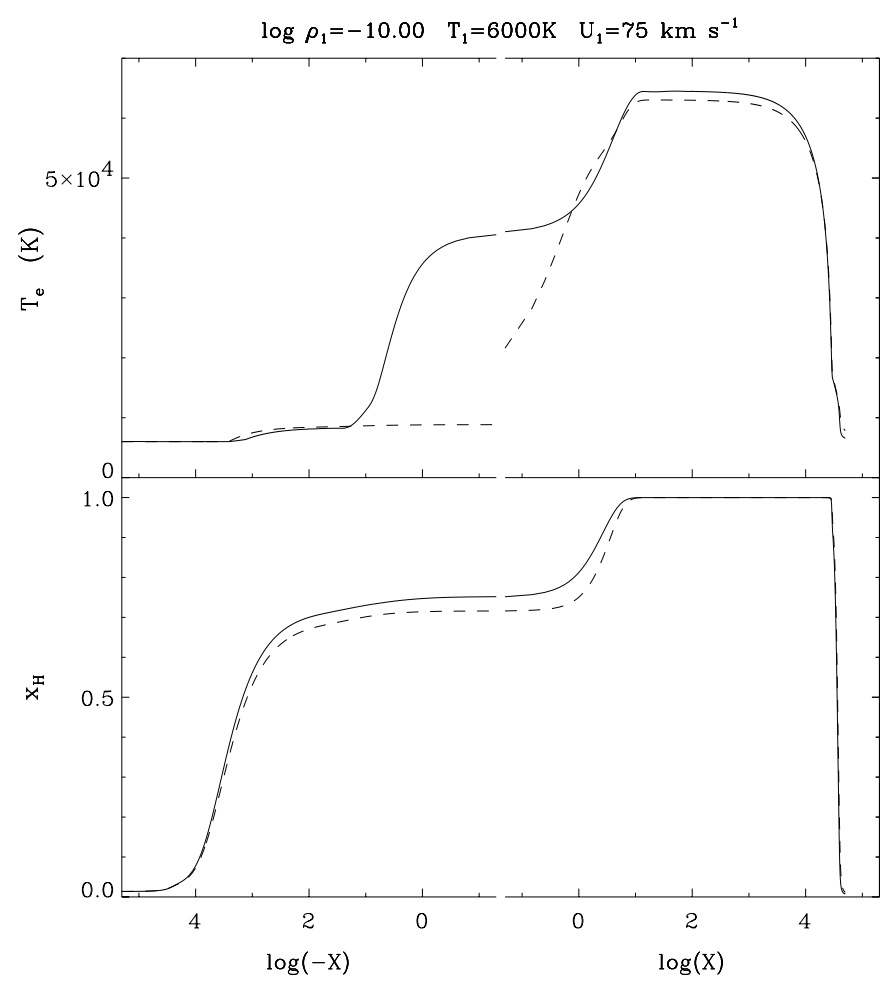

Fig. 4. The electron temperature $T_{\mathrm{e}}$ (upper panel) and the hydrogen ionization degree $x_{\mathrm{H}}$ (lower panel). In solid and dashed lines are represented the conductive and pure radiative models. The meaning of the left and the right panels is the same as in Fig. 3.

The efficiency of electron heat conduction in radiative shock waves depends on the hydrogen ionization degree ahead the discontinuous jump as well as on the electron temperature behind the discontinuous jump. Obviously, both these quantities increase with increasing upstream gas flow velocity $U_{1}$ though the preshock hydrogen ionization degree depends also on the temperature $T_{1}$ of the unperturbed ambient gas. In order to determine the threshold upstream velocity $U_{1}^{*}$ for electron thermal conduction we computed a number of models with different values of $T_{1}$ and $U_{1}$ but for the fixed value of the unperturbed gas density $\rho_{1}=10^{-10} \mathrm{gm} \mathrm{cm}^{-3}$. Results of these computations are summarized in Table 1 where the columns headed by $U_{1}, T_{1}$ and $M_{1}$ give the upstream gas flow velocity in $\mathrm{km} \mathrm{s}^{-1}$, the temperature of the unperturbed hydrogen gas and the upstream Mach number. The column headed by $x_{H}^{-}$gives the hydrogen ionization degree just ahead of the discontinuous jump of the shock wave model with electron heat conduction and the column headed by $\delta\left(x_{\mathrm{H}}^{-}\right)$gives the raise of the preshock hydrogen ionization degree in comparison with the pure radiative model. We found that the preshock raise of the hydrogen ionization degree due to effects of the electron heat conduction is $\delta\left(x_{\mathrm{H}}^{-}\right) \lesssim 0.05$. Obviously, $\delta\left(x_{\mathrm{H}}^{-}\right)=0$ not only in the weak shocks with negligible heat conduction but also in strong shocks with full ionization of the preshock hydrogen gas. In the latter case the excess of the radiation energy density in the Lyman continuum leads to the stronger radiative heating of the preshock gas.

In other to evaluate the role of electron thermal conduction in the structure of radiative shock waves we compare the
Table 1. Properties of radiative shock waves with electron heat conduction at $\rho_{1}=10^{-10} \mathrm{gm} \mathrm{cm}^{-3}$.

\begin{tabular}{rrrlllll}
\hline \hline$U_{1}$ & $T_{1}$ & $M_{1}$ & $x_{\mathrm{H}}^{-}$ & $\delta\left(x_{\mathrm{H}}^{-}\right)$ & $\varepsilon\left(\rho^{-}\right)$ & $\varepsilon\left(F_{\mathrm{Ly}, \mathrm{J}}\right)$ & $\varepsilon\left(\boldsymbol{F}_{\mathrm{R}}\right)$ \\
\hline 65 & 8000 & 5.2 & 0.84 & 0.02 & 1.04 & 1.19 & 1.14 \\
70 & 3000 & 10.9 & 0.47 & 0.01 & 1.02 & 1.08 & 1.03 \\
& 6000 & 7.7 & 0.73 & 0.02 & 1.05 & 1.21 & 1.16 \\
& 8000 & 5.6 & 1. & 0. & 1.08 & 1.30 & 1.28 \\
75 & 3000 & 11.7 & 0.62 & 0.02 & 1.03 & 1.13 & 1.07 \\
& 6000 & 8.2 & 0.75 & 0.04 & 1.04 & 1.20 & 1.14 \\
& 8000 & 6.0 & 1. & 0. & 1.10 & 1.33 & 1.30 \\
80 & 3000 & 12.5 & 0.86 & 0.05 & 1.09 & 1.38 & 1.32 \\
& 6000 & 8.8 & 0.98 & 0.01 & 1.06 & 1.25 & 1.20 \\
& 8000 & 6.4 & 1. & 0. & 1.08 & 1.27 & 1.26 \\
85 & 3000 & 13.2 & 1. & 0. & 1.10 & 1.30 & 1.26 \\
\hline
\end{tabular}

preshock gas density $\rho^{-}$, the Lyman continuum flux at the discontinuous jump $F_{\mathrm{Ly}, \mathrm{J}}$ and the total radiative flux emerging from the both boundaries of the shock wave model $\boldsymbol{F}_{\mathrm{R}}=$ $\frac{1}{2}\left(F_{\mathrm{R}, 1}+F_{\mathrm{R}, \mathrm{N}}\right)$ with corresponding quantities evaluated for the pure radiative models. To this end in the last three columns of Table 1 we list the ratios $\varepsilon\left(\rho^{-}\right)=\left(\rho^{-}\right)_{\mathrm{c}} /\left(\rho^{-}\right)_{\mathrm{r}}, \varepsilon\left(F_{\mathrm{Ly}, \mathrm{J}}\right)=$ $\left(F_{\text {Ly,J }}\right)_{\mathrm{c}} /\left(F_{\mathrm{Ly}, \mathrm{J}}\right)_{\mathrm{r}}$ and $\varepsilon\left(\boldsymbol{F}_{\mathrm{R}}\right)=\left(\boldsymbol{F}_{\mathrm{R}}\right)_{\mathrm{c}} /\left(\boldsymbol{F}_{\mathrm{R}}\right)_{\mathrm{r}}$.

As is seen from Table 1, the threshold upstream velocity is $U_{1}^{*} \approx 70 \mathrm{~km} \mathrm{~s}^{-1}$ and effects of electron thermal conduction become perceptible in strong shock waves with fully ionized hydrogen behind the discontinuous jump. At upstream gas flow velocities $U_{1} \geq 70 \mathrm{~km} \mathrm{~s}^{-1}$ the postshock electron temperature is high enough to support the necessary temperature gradient and at the same to ionize more than the half of the hydrogen atoms in the radiative precursor by the strong Lyman continuum flux emerging from the postshock region. Shown in Fig. 5 plots of the electron temperature in the vicinity of the discontinuous jump illustrate the very rapid growth of the conductive precursor with increasing upstream velocity $U_{1}$. Indeed, in the upstream velocity range $70 \mathrm{~km} \mathrm{~s}^{-1} \leq U_{1} \leq 85 \mathrm{~km} \mathrm{~s}^{-1}$ the additional radiative losses due to effects of electron heat conduction change from almost negligible values up to a quarter of the total radiative flux. Finally, as follows from Table 1, the higher temperature of the ambient gas significantly favours the electron heat conduction due to the higher ionization of the unperturbed ambient gas.

\section{Conclusion}

In this paper we have shown that in radiative shock waves with upstream velocities of $U_{1} \gtrsim 70 \mathrm{~km} \mathrm{~s}^{-1}$ the physical properties of the gas surrounding the discontinuous jump are sufficient for appearence of efficient electron heat conduction, the conductive flux being comparable with the Lyman continuum flux in the vicinity of the discontinuous jump. The existence of the narrow conductive precursor affects all the region of the Lyman continuum radiation trapped around the discontinuous jump between zones of preshock ionization and postshock recombination of the hydrogen gas. As a consequence, the hydrogen ionization degree increases in the radiative precursor by as much as $5 \%$ provided that the preshock hydrogen gas is partially ionized. 


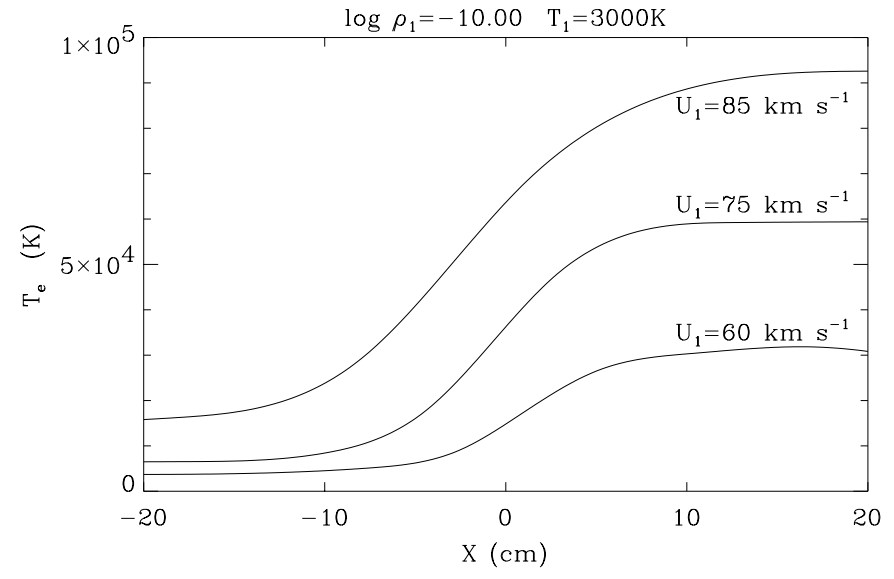

Fig. 5. The electron temperature $T_{\mathrm{e}}$ in the vicinity of the discontinuous jump in radiative shock waves with electron thermal conduction at upstream velocities $U_{1}=60,75$ and $85 \mathrm{~km} \mathrm{~s}^{-1}$.

The effect of the saturation of the electron conductive flux can be important only ahead of the discontinuity but for models considered in the present study we obtained $\left|\boldsymbol{F}_{\mathrm{e}}\right| \lesssim q_{\text {sat. }}$. The main conclusion of our study is that in shocks with velocities exceeding the threshold value $U_{1}^{*} \approx 70 \mathrm{~km} \mathrm{~s}^{-1}$ the electron thermal conduction significantly increases the radiative losses and, therefore, can diminish the efficiency of the shock-driven mass loss. More studies of radiative shock waves with electron heat conduction for wider ranges of $\rho_{1}, T_{1}$ and $U_{1}$ are needed.

Acknowledgements. The work of YAF has been done in part under the auspices of the Ministère de la Recherche Française as well as was supported in part by the Russian National Program "Astronomy" (item 1102). This paper is a part of the thesis of H. Le Coroller.

\section{References}

Belvedere, G., Chiuderi, C., \& Paterno, L. 1982, A\&A, 105, 133

Borkowski, K. J., \& Shull, J. M. 1990, ApJ, 348, 169

Borkowski, K. J., Shull, J. M., \& McKee, C. F. 1989, ApJ, 336, 979

Cowie, L. L., \& McKee, C. F. 1977, ApJ, 211, 135

Fadeyev, Y. A. 2001, Astron. Rep., 45, 361

Fadeyev, Y. A., \& Gillet, D. 1998, A\&A, 333, 687 (Paper I)

Fadeyev, Y. A., \& Gillet, D. 2000, A\&A, 354, 349 (Paper II)

Fadeyev, Y. A., \& Gillet, D. 2001, A\&A, 368, 901 (Paper III)

Grewal, M. S., \& Talbot, L. 1963, J. Fluid Mech., 16, 573

Imamura, J. N., Durisen, R. H., Lamb, D. Q., \& Weast, G. J. 1987, ApJ, 313, 298

Jaffrin, M. Y. 1965, Phys. Fluids, 8, 606

Lu, C. S., \& Huang, A. B. 1974, Phys. Fluids, 17, 1527

Mihalas, D., \& Weibel Mihalas, B. 1984, Foundations of radiation hydrodynamics (New York: Oxford University Press)

Nowak, T., \& Ulmschneider, P. 1977, A\&A, 60, 413

Spitzer, L. 1962, Physics of Fully Ionized Gases, 2nd edition (New York: Interscience)

Vidal, F., Matte, J. P., Casanova, M., \& Larroche, O. 1993, Phys. Fluids B, 5, 3182

Vidal, F., Matte, J. P., Casanova, M., \& Larroche, O. 1995, Phys. Plasmas, 2, 1412

Vinolo, A. R., \& Clarke, H. 1973, Phys. Fluids, 16, 1612

Zel'dovich, Y. B., \& Raizer, Y. P. 1967, Physics of shock waves and high-temperature hydrodynamic phenomena (New York: Academic Press), ed. W. D. Hayes, \& R. F. Probstein 